\title{
Single-beam spectrally controlled two-dimensional Raman spectroscopy
}

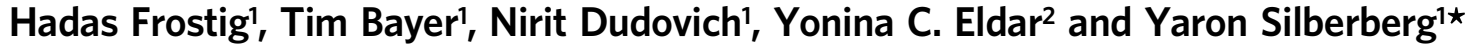

\begin{abstract}
Vibrational modes are often localized in certain regions of a molecule, and so the coupling between these modes is sensitive to the molecular structure. Two-dimensional vibrational spectroscopy can probe the strength of this coupling in a manner analogous to two-dimensional NMR spectroscopy, but on ultrafast timescales. Here, we demonstrate how twodimensional Raman spectroscopy, based on fifth-order optical nonlinearity, can be performed with a single beam of shaped femtosecond optical pulses. Our spectroscopy scheme offers not only a major simplification of the conventional set-up, but also an inherent elimination of a competing nonlinear signal, which overwhelms the desired signal in other schemes and carries no coupling information.
\end{abstract}

U nderstanding the structure of complex molecular systems and following their evolution during various chemical and biological processes are central issues in chemical physics and biophysics. One well-established method for extracting structural information is two-dimensional NMR spectroscopy, which utilizes correlation measurements between various resonances to determine their connectivity or relative distance. However, the relatively long timescales of radiofrequency (RF) pulses limit the technique's temporal resolution to milliseconds, far above the timescale necessary to follow the time dynamics of liquids and therefore many biological materials. This limitation can be overcome by using two-dimensional optical spectroscopies, which make use of femtosecond pulses to examine the coupling between energy levels ${ }^{1-8}$. Two-dimensional infrared spectroscopy has been used successfully to examine the structural dynamics of a variety of samples using vibrational coupling, including peptides and $\mathrm{DNA}^{9-12}$. Nevertheless, the low-frequency regime, which is of particular interest because it includes the collective motions of large molecules and molecular complexes, is beyond the range of two-dimensional-infrared spectroscopy and not yet accessible with two-dimensional terahertz methods.

Fifth-order two-dimensional impulsive Raman spectroscopy can probe the low-frequency regime using ultrashort pulses in the visible range, and therefore attracted a great deal of interest when introduced $^{13-17}$. The conventional scheme makes use of five femtosecond pulses in a non-collinear geometry. The pulses arrive at three distinct times to the sample and consecutively excite, couple and probe vibrational levels via impulsive Raman processes (Fig. 1a). The non-collinear geometry is usually used to separate the fifthorder field $E^{(5)}$ from the third-order field $E^{(3)}$ and the excitation light, and to satisfy phase-matching requirements. However, from the start the use of two-dimensional Raman spectroscopy was hindered by unwanted lower-order cascade signals, which often overwhelmed the desired signal completely ${ }^{18-20}$. This difficulty, together with the complex multi-beam set-up required, prevented two-dimensional Raman spectroscopy from developing into a widely used spectroscopic technique.

In this Article we present a scheme that uses spectral shaping techniques to excite and probe this entire process with a single femtosecond pulse. We use two novel elements. The first is coherent control of the excitation and coupling processes, which encodes the multi-beam set-up into spectral properties of the pulse and thus considerably simplifies the experimental apparatus. The second is spectrally resolved detection, which eliminates the unwanted cascade signal.

\section{Principle}

Control of the excitation process is achieved by applying a periodic spectral phase modulation with a period $\Omega$ to the input pulse spectrum using a pulse-shaper ${ }^{21-24}$. This creates a pulse train with a delay $\tau=2 \pi / \Omega$ in the time domain. The energy delivered to the molecule by the train adds coherently only for vibrational levels that complete an integer number of oscillations during $\tau$. When this condition is obeyed, stimulated Raman gain occurs on the red side of the pulse spectrum, and stimulated Raman loss occurs on the blue side. The overall process results in a redshift of the output spectrum that is proportional to the amount of excitation of the Raman level ${ }^{25}$. One-dimensional single-beam stimulated Raman spectroscopy can thus be performed by scanning the modulation period $\Omega$ and measuring the amount of spectral redshift (see diagram of set-up in Fig. 1b). The Fourier transform of the recorded spectral shift yields the Raman spectrum. The results of performing one-dimensional single-beam stimulated Raman spectroscopy on several liquid samples are presented in Fig. 2.

The extension to two-dimensional spectroscopy is relatively straightforward and requires no modification of the set-up. The shaper is now used to apply a spectral phase mask that is the sum of two periodic phase functions with periods $\Omega_{01}$ and $\Omega_{12}$. The resulting pulse sequence in the time domain is a convolution of two trains, one with delay $\tau_{01}=2 \pi / \Omega_{01}$ and the other with delay $\tau_{12}=2 \pi / \Omega_{12}$ (Fig. 3a). Each pulse in the resulting sequence is both a member of a train in which the pulse-to-pulse separation is $\tau_{01}$, and a member of another train in which the separation is $\tau_{12} . \tau_{01}$ trains selectively excite molecules from the ground state to vibrational level $\omega_{1}$, whereas $\tau_{12}$ trains selectively couple from level $\omega_{1}$ to another vibrational level $\omega_{2}$. Thus, by carefully choosing $\Omega_{01}$ and $\Omega_{12}$ we can control which Raman level is excited and to which level the energy is coupled. To perform two-dimensional spectroscopy, the two modulation periods are scanned and the resulting two-dimensional data are either Fourier-transformed or analysed with an advanced signal analysis method-block 


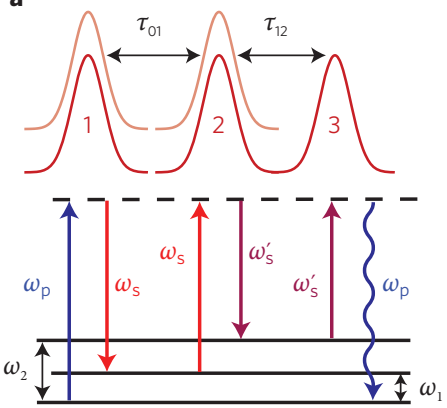

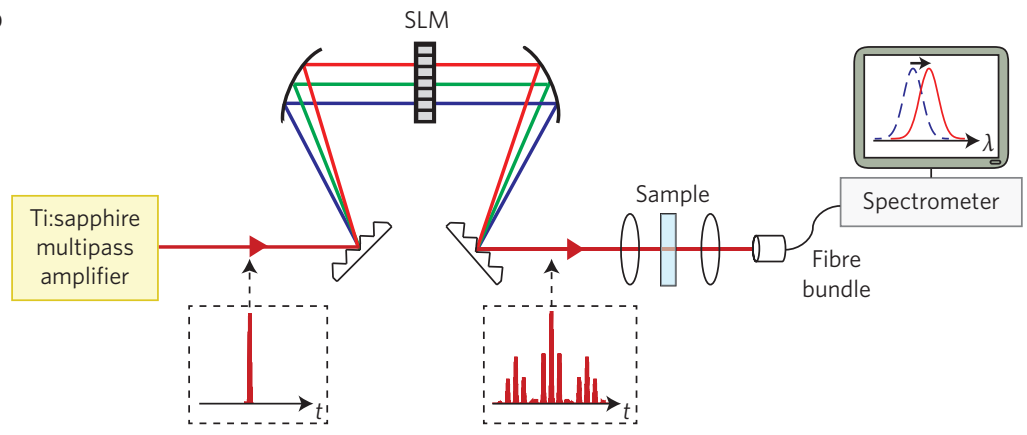

Figure 1 | Two-dimensional Raman spectroscopy: principle and single-beam implementation. a, Energy diagram of the fifth-order Raman process. Conventionally, this process is excited with the pulse sequence shown at the top, which is composed of five pulses in a non-collinear geometry. The pulses arrive at three distinct times at the sample, causing mode excitation, mode-to-mode coupling and probing of the vibrational levels, respectively. $\mathbf{b}$, Schematic of the set-up of single-beam spectrally controlled Raman spectroscopy, which uses a single shaped pulse to excite the entire fifth-order process. The same set-up is used for both one-dimensional and two-dimensional spectroscopy schemes. The pulses are phase-shaped using a spatial light modulator (Fig. 3) and then focused onto the liquid samples. The spectral shift of the transmitted light is measured with a spectrometer.

orthogonal matching pursuit (BOMP) $)^{26,27}$-which can be utilized to extract spectroscopic information (see below).

At first glance, the collinearity of our set-up seems to have greatly simplified the experimental apparatus yet obscured the detected signal. At the output, we detect the coherent interference of the input, third-order and fifth-order fields. The most significant terms of this interference are:

$$
\begin{aligned}
I_{\text {total }}(\lambda) & =\left|E_{\text {in }}(\lambda)+E^{(3)}(\lambda)+E^{(5)}(\lambda)\right|^{2} \\
& \cong I_{\text {in }}+2\left|E_{\text {in }}\right|\left|E^{(3)}\right| \cos \phi_{3}+I^{(3)}+2\left|E_{\text {in }}\right|\left|E^{(5)}\right| \cos \phi_{5}
\end{aligned}
$$

where $E_{\text {in }}(\lambda)$ is the input field as a function of wavelength, $E^{(3)}$ and $E^{(5)}$ are the third- and fifth-order nonlinear fields, respectively, $\phi_{3}$ and $\phi_{5}$ are their spectral phases with respect to $E_{\text {in }}$ and $\left|E_{\text {in }}\right| \gg\left|E^{(3)}\right| \gg\left|E^{(5)}\right|$. The coupling information is contained in the last term, $2\left|E_{\text {in }}\right|\left|E^{(5)}\right| \cos \phi_{5}$, which is smaller than the other terms. Nevertheless, because we are probing resonant vibrational levels, all four terms behave differently for a two-dimensionally
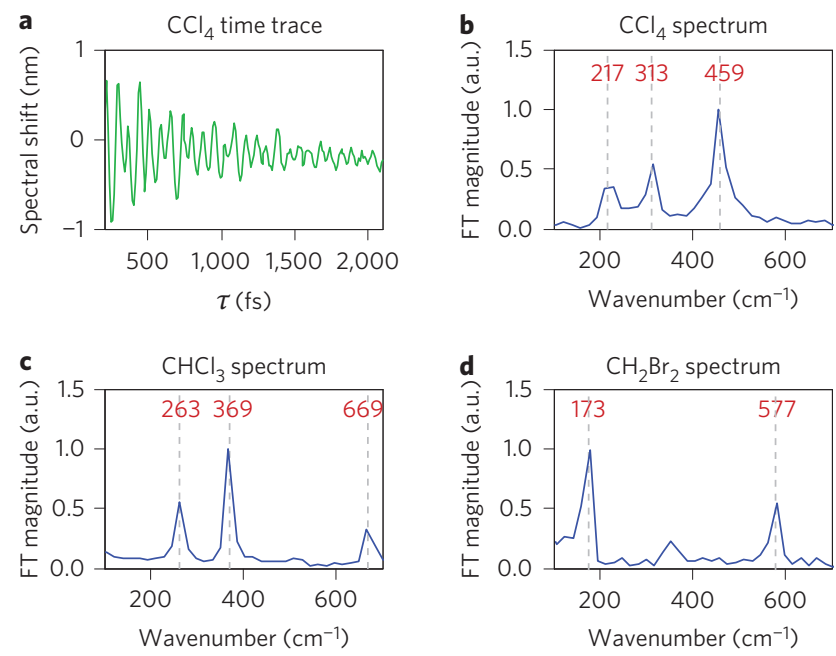

Figure 2 | One-dimensional single-beam spectrally controlled stimulated Raman spectroscopy: experimental results. a Measured spectral shift of the output pulse as a function of train pulse-to-pulse delay $\tau$ for carbon tetrachloride $\left(\mathrm{CCl}_{4}\right)$. b. Fourier transform (FT) of the trace in $\mathbf{a}$, which shows the Raman spectrum of $\mathrm{CCl}_{4}$. $\mathbf{c}$, Measured spectrum of chloroform $\left(\mathrm{CHCl}_{3}\right)$. d, Measured spectrum of dibromomethane $\left(\mathrm{CH}_{2} \mathrm{Br}_{2}\right)$. The known Raman lines are shown above for reference. varying delay. The first term remains constant, regardless of the applied spectral phase, while the second and third terms oscillate with the same frequency in both $\tau_{01}$ and $\tau_{12}$ and thus appear as peaks on the diagonal of a two-dimensional Fourier transform plot. Only the term $2\left|E_{\text {in }}\right|\left|E^{(5)}\right| \cos \phi_{5}$ has components that oscillate with one frequency in $\tau_{01}$ and another in $\tau_{12}$, causing off-diagonal peaks. The coupling information can therefore be extracted readily from the off-diagonal information of the two-dimensional spectrum.

As mentioned above, a central issue in conventional twodimensional Raman spectroscopy is the presence of an unwanted lower-order cascaded signal. The total fifth-order response includes two components: the direct field, $E_{\mathrm{dir}}^{(5)}$, which carries information about the intramolecular vibrational coupling, and the cascade field, $E_{\text {cas }}^{(5)}$. $E_{\text {cas }}^{(5)}$ is the result of two consecutive thirdorder interactions with two different molecules, where the output field from the first molecule serves as the input to the second. Thus $E_{\text {cas }}^{(5)}$ contains only one-dimensional molecular data, yet appears as off-diagonal peaks just like $E_{\text {dir }}^{(5)}$ when the two third-order interactions are with different vibrational levels. Several methods have been developed to reduce the cascade field, including specially tailored five-directional wavevector matching geometries ${ }^{15}$, polarization control ${ }^{28}$ and adding local oscillator fields used for heterodyne detection ${ }^{29-31}$.

Our use of a collinear geometry together with spectral-shift measurements inherently amplifies $E_{\text {dir }}^{(5)}$ and suppresses $E_{\text {cas }}^{(5)}$. The reason for this selective amplification is that $E_{\text {in }}$ serves, in practice, as a local oscillator that amplifies the component of the fifth-order field that is in phase with it, typically by several orders of magnitude. Therefore, for real $E_{\mathrm{in}}$, only the real parts of both $E_{\mathrm{dir}}^{(5)}$ and $E_{\text {cas }}^{(5)}$ are detected $\left(\cos \phi_{5}=0\right.$ for the imaginary part of $E^{(5)}$ in equation (1)). Furthermore, it is known that the $n$ th-order nonlinear polarization $P^{(n)}$ generates a field, $E^{(n)} \propto i P^{(n)}$. That is, every radiation event adds a $\pi / 2$ phase to the field. Because the field radiated by the direct fifth-order polarization undergoes one radiation event whereas the field radiated by the cascaded third-order polarization undergoes two such events, $E_{\text {cas }}^{(5)}$ and $E_{\text {dir }}^{(5)}$ are very similar except that their real and imaginary parts are swapped, as can be observed in Fig. $3 \mathrm{c}$ and d. Whereas the in-phase component of $E_{\text {dir }}^{(5)}$ is antisymmetric (the real part in Fig. 3c) and therefore causes a blueshift when combined with the stronger $E_{\mathrm{in}}$, the in-phase component of $E_{\mathrm{cas}}^{(5)}$ is spectrally symmetric (the real part in Fig. 3d), and therefore leaves the spectrum unshifted. Consequently, spectral shifts are insensitive to the cascade signal. Although previous realizations of cascade suppression in a non-collinear geometry used a local oscillator ${ }^{29-31}$, those methods were still sensitive to the cascade signal from resonant vibrational levels as they relied solely on the field quadrature of the signal. 

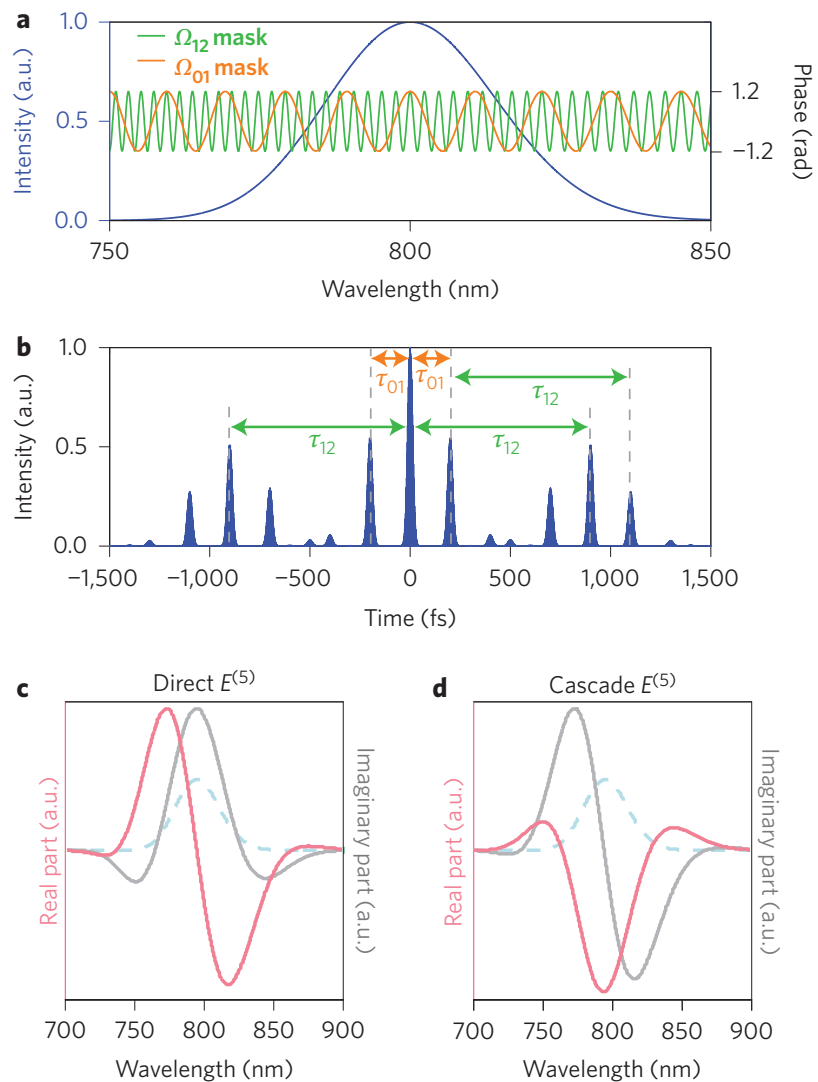

Figure 3 | Spectral control and spectrally-resolved detection of the fifthorder Raman process. $\mathbf{a}$, The sum of two periodic phase masks, one with period $\Omega_{01}$ (orange) and the other with period $\Omega_{12}$ (green), is applied to the incoming pulse spectrum (blue). $\mathbf{b}$, The resulting pulse sequence in the time domain is a convolution of two trains: one with a pulse-to-pulse delay of $\tau_{01}$ and one with a delay of $\tau_{12}$. Each pulse in the resulting sequence is both a member of a train in which the pulse-to-pulse separation is $\tau_{01}$ and a member of another train in which the separation is $\tau_{12}$. $\mathbf{c}$. Spectrum of the real (pink) and imaginary (grey) parts of the direct fifth-order field $E_{\text {dir }}^{(5)}$ and the input field $E_{\text {in }}$ (dashed line, not to scale). The real part, which is detected, will cause a spectral shift when interfered with the input field. d, As in c, but for the cascade fifth-order field, $E_{\text {cas }}^{(5)}$ (parallel and sequential mechanisms ${ }^{18}$ ). The detected real part will not cause a spectral shift.

\section{Results}

Two-dimensional spectra computed from a Fourier transform. The single-beam two-dimensional Raman spectra of several liquid samples are shown in Fig. 4. The measured two-dimensional spectrum of $\mathrm{CCl}_{4}$ is presented in Fig. 4a. Strong peaks corresponding to the one-dimensional data are present on the diagonal. We also observe off-diagonal peaks of each mode with its overtones and undertones, which are caused by the onedimensional $E^{(3)}$ signal as well. Several cross-peaks are clearly visible. A cross-peak between the $v_{1}$ and $v_{2}$ modes $\left(459 \mathrm{~cm}^{-1}\right.$ and $\left.217 \mathrm{~cm}^{-1}\right)$ and a cross-peak between the $v_{1}$ and $v_{4}$ modes $\left(459 \mathrm{~cm}^{-1}\right.$ and $313 \mathrm{~cm}^{-1}$ ) are noticeable, whereas the coupling between the $v_{4}$ and $v_{2}$ modes $\left(313 \mathrm{~cm}^{-1}\right.$ and $\left.217 \mathrm{~cm}^{-1}\right)$ is less significant. The measured two-dimensional spectrum of $\mathrm{CHCl}_{3}$ is presented in Fig. 4b. Note that the molecular couplings in $\mathrm{CHCl}_{3}$ are somewhat weaker and there is only some coupling between the $v_{6}$ and $v_{3}$ modes $\left(263 \mathrm{~cm}^{-1}\right.$ and $\left.369 \mathrm{~cm}^{-1}\right)$. To test whether the scheme is truly insensitive to the cascade signal, the two-dimensional spectrum of a $\mathrm{CCl}_{4}: \mathrm{CHCl}_{3}$ mixture (1:1 mole ratio) was measured. The result is presented in Fig. 4c. Note that the cross-peaks between modes of the same molecular (for example, between the $459 \mathrm{~cm}^{-1}$
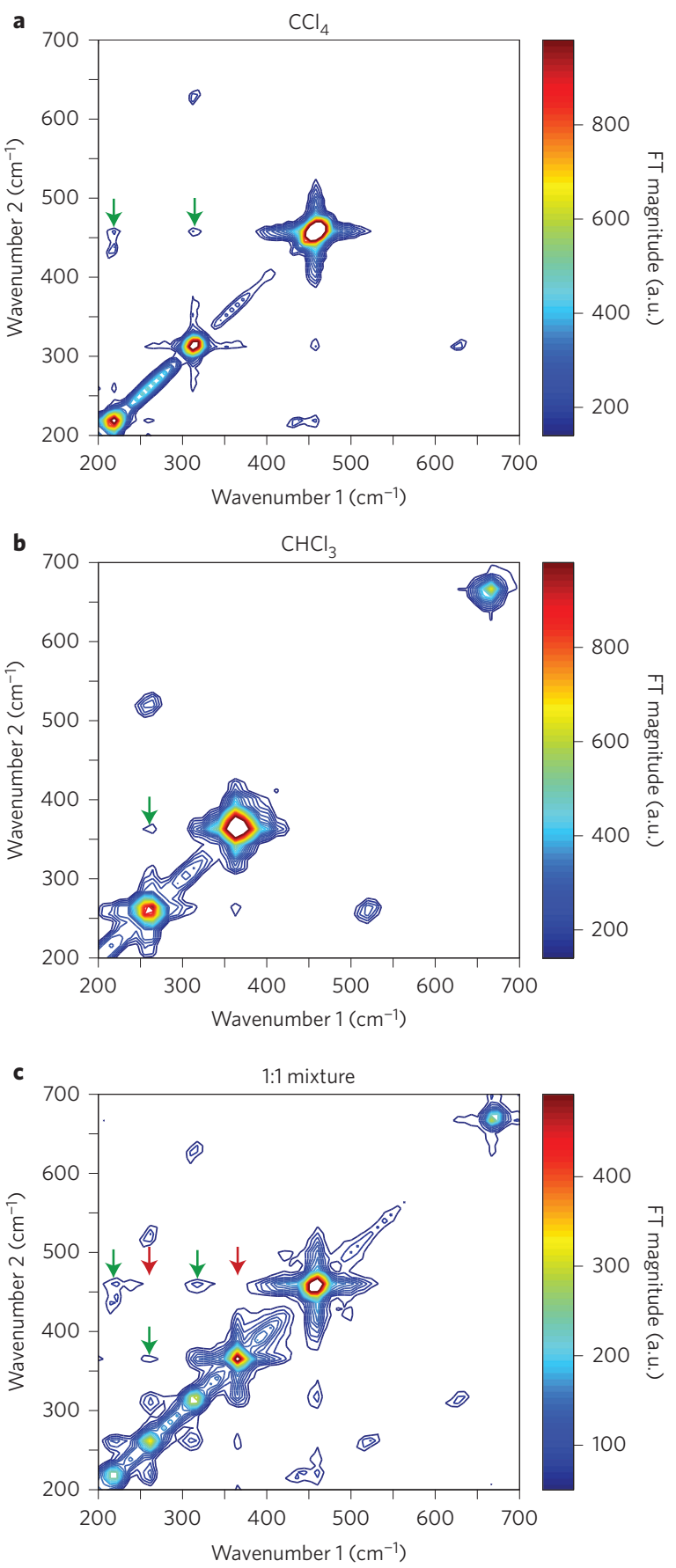

Figure 4 | Two-dimensional single-beam spectrally controlled Raman spectroscopy: experimental results. a, Two-dimensional spectrum of $\mathrm{CCl}_{4}$. In addition to the strong diagonal peaks, cross-peaks between the $459 \mathrm{~cm}^{-1}$ and $217 \mathrm{~cm}^{-1}$ modes and between the $459 \mathrm{~cm}^{-1}$ and $313 \mathrm{~cm}^{-1}$ modes are observed (marked with arrows). b, Two-dimensional spectrum of $\mathrm{CHCl}_{3}$. Some coupling is observed between the $263 \mathrm{~cm}^{-1}$ and $369 \mathrm{~cm}^{-1}$ modes. c, Two-dimensional spectrum of a 1:1 mole ratio $\mathrm{CCl}_{4}: \mathrm{CHCl}_{3}$ mixture. Significant cross-peaks are observed between the modes of the same molecule (marked with green arrows), such as between the $313 \mathrm{~cm}^{-1}$ and $459 \mathrm{~cm}^{-1}$ modes of $\mathrm{CCl}_{4}$, but not between the modes of different molecules (locations marked with red arrows), such as between the $459 \mathrm{~cm}^{-1}$ mode of $\mathrm{CCl}_{4}$ and the $369 \mathrm{~cm}^{-1}$ mode of $\mathrm{CHCl}_{3}$.

and $313 \mathrm{~cm}^{-1}$ modes of $\mathrm{CCl}_{4}$, and between the $263 \mathrm{~cm}^{-1}$ and $369 \mathrm{~cm}^{-1}$ modes of $\mathrm{CHCl}_{3}$ ) are present, whereas cross-peaks between modes of the two different molecular species are not 

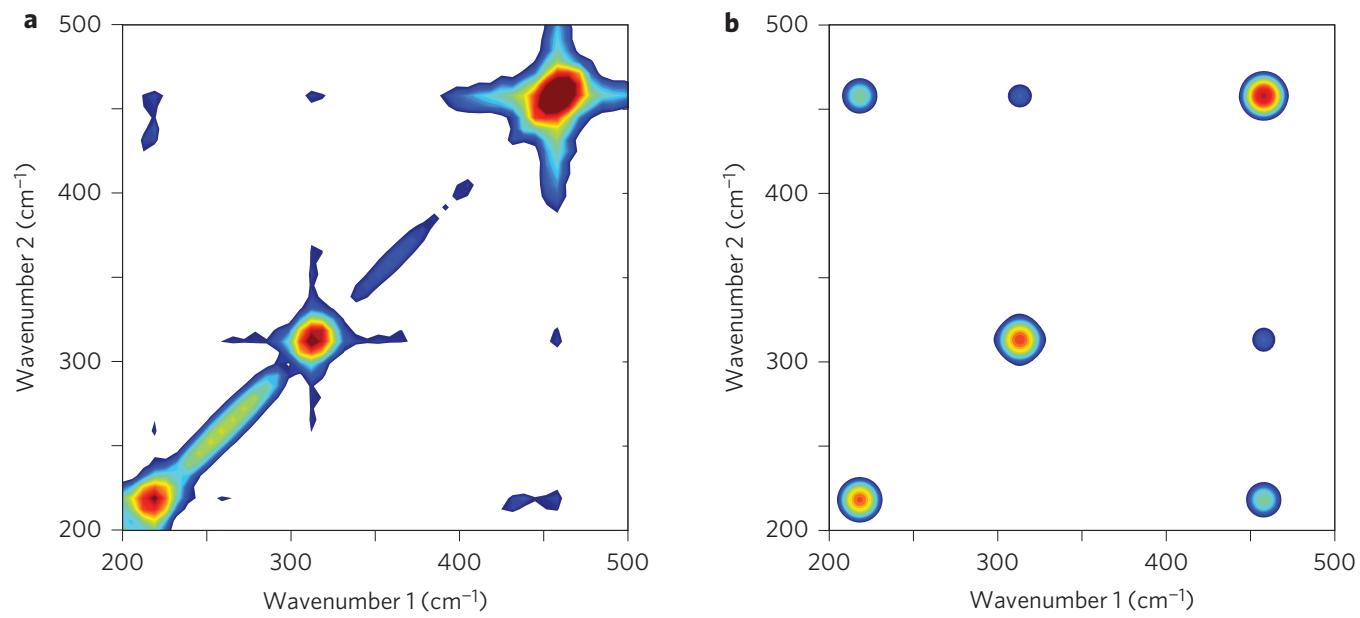

Figure 5 | Comparison of the two signal-processing methods used to extract cross-peak information. $\mathbf{a}$, Two-dimensional spectrum of $\mathrm{CCl}_{4,}$, produced with a discrete Fourier transform. $\mathbf{b}$, Clean two-dimensional spectrum of $\mathrm{CCl}_{4}$, computed using BOMP analysis. The long tails of the diagonal peaks that may artificially increase cross-peak values were successfully eliminated, as well as other irrelevant off-diagonal peaks. Both contour plots are presented in a log scale.

observed (for example, the lack of a cross-peak between the $459 \mathrm{~cm}^{-1}$ and $369 \mathrm{~cm}^{-1}$ modes or between the $459 \mathrm{~cm}^{-1}$ and $263 \mathrm{~cm}^{-1}$ modes). This result indicates that the signal measured by our scheme does in fact originate from intramolecular coupling.

Analysing time-domain signals with BOMP. Some ambiguity may arise in the two-dimensional Fourier analysis of the type presented above due to the broadened shape of the diagonal peaks. The broadening is caused both by homogeneous broadening of the molecular lineshape and by spectral leakage. Spectral leakage is a phenomenon that occurs in discrete Fourier transforms (DFT) owing to the limited temporal window of the measurements. As a result of both mechanisms, the strong diagonal peaks appear to have long vertical and horizontal tails. These tails may combine at the crossing point between a horizontal tail of one line and a vertical tail of another, artificially increasing the value of the cross-peak. To eliminate this ambiguity, the time traces were reanalysed using BOMP, as proposed by Eldar and co-authors ${ }^{26,27}$. BOMP is an iterative procedure that identifies and removes the strongest component of the signal at each iteration. Using BOMP, the signal caused by the first three terms in equation(1) was removed from the total measured signal, and the residual was fitted to obtain the amplitudes and lineshapes of the true crosspeak signal. The entire procedure was performed in the time domain to ensure that energy leaks from the diagonal peaks were not present. Due to the nature of compressed sensing techniques, the analysis with BOMP also provides significantly higher spectral resolution than the DFT, approximately an order of magnitude better than the Nyquist limit ${ }^{32}$. The BOMP analysis procedure will be explained in more detail in a future publication.

The reconstructed signal of the experimental $\mathrm{CCl}_{4}$ measurement, computed with BOMP analysis, is presented in Fig. 5b. Note that the irrelevant off-diagonal peaks caused by overtones have been eliminated, as well as the long tails of the diagonal peaks. Consequently, we can extract background-free quantitative information about the strengths of the cross-peaks from the results. From the BOMP analysis we find that the cross-peak between the $v_{1}$ and $v_{2}$ modes $\left(459 \mathrm{~cm}^{-1}\right.$ and $\left.217 \mathrm{~cm}^{-1}\right)$ is strongest, about four times stronger than that of the $v_{1}$ and $v_{4}$ modes, as expected due to the Fermi resonance between $2 v_{2}$ and $v_{1}$.

\section{Discussion}

We have demonstrated the acquisition of two-dimensional fifth-order Raman spectra through the use of a single, phase-shaped ultrashort pulse. Our technique inherently eliminates the cascade signal, making the vibrational coupling information easy to extract. The experimental apparatus is collinear and requires only a simple imaging system and a pulse-shaper, which is now commercially available. The spectral shaping approach can easily be extended to create more complex temporal fields, including sequences of pulses with different polarizations and spectral content. In particular, timeresolved coupling measurements, as well as $\mathrm{N}$-dimensional spectroscopy, can be readily implemented by creating an $N$-pulse sequence using phase and amplitude shaping.

An important advantage of using BOMP analysis, in addition to the removal of the background in the cross-peak locations, is that it significantly enhances the spectral resolution of our method. Thus, although the resolution of the DFT plots is limited by the shaping resolution to $\sim 6-7 \mathrm{~cm}^{-1}$, the resolution of the plots produced by BOMP is below $1 \mathrm{~cm}^{-1}$. Simple upgrades of our shaper can also significantly increase the resolution of the DFT plots.

The present system is suitable for studying a large frequency span, from below $100 \mathrm{~cm}^{-1}$ to above $1,000 \mathrm{~cm}^{-1}$. The lower end of this span, which cannot currently be reached with other two-dimensional methods, is particularly important for studying large biomolecules and molecular complexes. In our previous study of single-pulse stimulated Raman scattering spectroscopy lines as low as $51 \mathrm{~cm}^{-1}$ were resolved ${ }^{25}$, indicating that the spectral range of our two-dimensional method can extend further down to several tens of wavenumbers. Moreover, the use of multi-pulse trains reduces the pulse peak intensity significantly, allowing for larger input pulse energies to be used without causing damage to the sample. Finally, the heterodyned nature of our detection results in a large amplification of the fifthorder signal. All these features, in addition to the simple collinear geometry of our set-up, suggest potential use in biological samples. To that end, the detection limit of our method can be improved considerably by integrating lock-in detection, which can filter out noise due to the instability of the input pulse power.

The simplicity and technical capabilities of our method overcome some of the major hurdles that have previously restricted the use of two-dimensional Raman spectroscopy. This opens the door to or perhaps: paves the way towards a broad range of applications of this technique, including spatially resolved measurements of molecular structure in biological samples.

\section{Methods}

The spectral phase of $\sim 25$ fs pulses centred at $795 \mathrm{~nm}$ (pulse energies of $100 \mathrm{~nJ}-1 \mu \mathrm{J}$ ) was controlled using a pulse shaper with a computer-controlled liquid-crystal spatial 
light modulator (Jenoptik SLM-S640) at the Fourier plane. The shaper was also used to compensate for the spectral phase acquired due to the optics in the set-up. Applied masks for two-dimensional spectroscopy were of the form $\phi(f)=1.2\left[\cos \left(2 \pi \tau_{01} f\right)+\cos \left(2 \pi \tau_{12} f\right)\right]$. The shaped pulses were focused by a $15 \mathrm{~cm}$ lens onto a quartz cell (path length of $1 \mathrm{~cm}$ ) filled with the liquid samples, and the transmitted light was coupled into a spectrometer. The mean of the recorded spectrum was computed for each phase mask and the spectral shift was calculated with respect to a reference measurement. Finally, the spectral shift as a function of $\tau_{01}$ and $\tau_{12}$ was Fourier-transformed or analysed with BOMP to obtain the Raman spectrum of the sample. Because the phase masks used in this scheme are cosine functions, the time-domain signal is insensitive to the sign of $\tau_{01}$ and $\tau_{12}$. Thus, the signal measured for the $\tau_{01}, \tau_{12}>0$ quadrant can be reflected to fill in the signal for the other quadrants. We used this procedure on our data before Fouriertransforming in order to increase the spectral resolution. Although the original data contain both the real and imaginary parts of the spectra, using this reflection procedure will yield only their real part. Using BOMP analysis, however, retrieves both the real and imaginary parts of the spectra.

\section{Received 30 September 2014; accepted 18 March 2015;} published online 20 April 2015

\section{References}

1. Hybl, J. D., Albrecht Ferro, A. \& Jonas, D. M. Two-dimensional Fourier transform electronic spectroscopy. J. Chem. Phys. 115, 6606-6622 (2001).

2. Brixner, T. et al. Two-dimensional spectroscopy of electronic couplings in photosynthesis. Nature 434, 625-628 (2005).

3. Tian, P., Keusters, D., Suzaki, Y. \& Warren, W. S. Femtosecond phase-coherent two-dimensional spectroscopy. Science 300, 1553-1555 (2003).

4. Grumstrup, E. M., Shim, S. H., Montgomery, M. A., Damrauer, N. H. \& Zanni, M. T. Facile collection of two-dimensional electronic spectra using femtosecond pulse-shaping technology. Opt. Express 15, 16681-16690 (2007).

5. Hornung, T., Vaughan, J. C., Feurer, T. \& Nelson, K. A. Degenerate four-wave mixing spectroscopy based on two-dimensional femtosecond pulse shaping. Opt. Lett. 29, 2052-2054 (2004).

6. Molesky, B. P., Giokas, P. G., Guo, Z. \& Moran, A. M. Multidimensional resonance Raman spectroscopy by six-wave mixing in the deep UV. J. Chem. Phys. 141, 114202 (2014).

7. Glenn, R. \& Mukamel, S. Multidimensional spectroscopy with a single broadband phase-shaped laser pulse. J. Chem. Phys. 140, 144105 (2014).

8. Baiz, C. R., Schach, D. \& Tokmakoff, A. Ultrafast 2D IR microscopy. Opt. Express 22, 18724-18735 (2014).

9. Kolano, C., Helbing, J., Kozinski, M., Sander, W. \& Hamm, P. Watching hydrogen-bond dynamics in a beta-turn by transient two-dimensional infrared spectroscopy. Nature 444, 469-472 (2006).

10. Asplund, M. C., Zanni, M. T. \& Hochstrasser, R. M. Two-dimensional infrared spectroscopy of peptides by phase-controlled femtosecond vibrational photon echoes. Proc. Natl Acad. Sci. USA 97, 8219-8224 (2000).

11. Krummel, A. T. \& Zanni, M. T. DNA vibrational coupling revealed with two-dimensional infrared spectroscopy: insight into why vibrational spectroscopy is sensitive to DNA structure. J. Phys. Chem. B 110, 13991-14000 (2006).

12. Fayer, M. D. Fast protein dynamics probed with infrared vibrational echo experiments. Annu. Rev. Phys. Chem. 52, 315-356 (2001).

13. Tanimura, Y. \& Mukamel, S. Two-dimensional femtosecond vibrational spectroscopy of liquids. J. Chem. Phys. 99, 9496-9511 (1993).

14. Tokmakoff, A., Lang, M. J., Larsen, D. S. \& Fleming, G. R. Two-dimensional Raman spectroscopy of vibrational interactions in liquids. Phys. Rev. Lett. 79, 2702-2705 (1997).

15. Blank, D. A., Kaufman, L. J. \& Fleming, G. R. Direct fifth-order electronically nonresonant Raman scattering from $\mathrm{CS}_{2}$ at room temperature. J. Chem. Phys. 113, 771-778 (2000).
16. Mukamel, S., Piryatinski, A. \& Chernyak, V. Two-dimensional Raman echoes: femtosecond view of molecular structure and vibrational coherence. Acc. Chem. Res. 32, 145-154 (1999).

17. Okumura, K., Tokmakoff, A. \& Tanimura, Y. Structural information from two-dimensional fifth-order Raman spectroscopy. J. Chem. Phys. 111, 492-503 (1999).

18. Ulness, D. J., Kirkwood, J. C. \& Albrecht, A. C. Competitive events in fifth order time resolved coherent Raman scattering: direct versus sequential processes. J. Chem. Phys. 108, 3897-3902 (1998).

19. Blank, D. A., Kaufman, L. J. \& Fleming, G. R. Fifth-order two-dimensional Raman spectra of $\mathrm{CS}_{2}$ are dominated by third-order cascades. J. Chem. Phys. 111, 3105-3114 (1999).

20. Wilson, K. C., Lyons, B., Mehlenbacher, R., Sabatini, R. \& McCamant, D. W. Two-dimensional femtosecond stimulated Raman spectroscopy: observation of cascading Raman signals in acetonitrile. J. Chem. Phys. 131, 214502 (2009).

21. Dudovich, N., Oron, D. \& Silberberg, Y. Single-pulse coherently controlled nonlinear Raman spectroscopy and microscopy. Nature 418, 512-514 (2002).

22. Dudovich, N., Oron, D. \& Silberberg, Y. Single-pulse coherent anti-Stokes Raman spectroscopy in the fingerprint spectral region. J. Chem. Phys. 118, 9208-9215 (2003).

23. Jang, H. U. et al. Interaction of a finite train of short pulses with an atomic ladder system. Phys. Rev. A 82, 043424 (2010).

24. Pestov, D., Lozovoy, V. V. \& Dantus, M. Multiple independent comb shaping (MICS): phase-only generation of optical pulse sequences. Opt. Express 17, 14351-14361 (2009).

25. Frostig, H., Katz, O., Natan, A. \& Silberberg, Y. Single-pulse stimulated Raman scattering spectroscopy. Opt. Lett. 36, 1248-1250 (2011).

26. Duarte, M. F. \& Eldar, Y. C. Structured compressed sensing: from theory to applications. IEEE Trans. Signal Process. 59, 4053-4085 (2011).

27. Eldar, Y. C., Kuppinger, P. \& Bolcskei, H. Block-sparse signals: uncertainty relations and efficient recovery. IEEE Trans. Signal Process. 58, 3042-3054 (2010).

28. Kaufman, L. J., Blank, D. A. \& Fleming, G. R. Polarization selectivity in fifth-order electronically nonresonant Raman scattering from $\mathrm{CS}_{2}$. J. Chem. Phys. 114, 2312-2331 (2001).

29. Golonzka, O., Demirdoven, N., Khalil, M. \& Tokmakoff, A. Separation of cascaded and direct fifth-order Raman signals using phase-sensitive intrinsic heterodyne detection. J. Chem. Phys. 113, 9893-9896 (2000).

30. Cho, M. et al. Intrinsic cascading contributions to the fifth- and seventh-order electronically off-resonant Raman spectroscopies. J. Chem. Phys. 112, 2082-2094 (2000).

31. Astinov, V., Kubarych, K. J., Milne, C. J. \& Miller, R. J. D. Diffractive optics based two-color six-wave mixing: phase contrast heterodyne detection of the fifth order Raman response of liquids. Chem. Phys. Lett. 327, 334-342 (2000).

32. Dunbar, J. A., Osborne, D. G., Anna, J. M. \& Kubarych, K. J. Accelerated 2D-IR using compressed sensing. J. Phys. Chem. Lett. 4, 2489-2492 (2013).

\section{Acknowledgements}

The authors thank B. Bruner and L. Chuntonov for useful discussions. This work was supported by Icore (Israeli centres of research excellence of the ISF), the Crown Photonics Center, the Wolfson Foundation and the European ICT project FAMOS.

\section{Author contributions}

H.F., T.B., N.D. and Y.S. designed the experiment, analysed the data and prepared the manuscript. H.F. and T.B. performed the experiment. Y.C.E. guided the BOMP analysis.

\section{Additional information}

Reprints and permissions information is available online at www.nature.com/reprints. Correspondence and requests for materials should be addressed to Y.S.

\section{Competing financial interests}

The authors declare no competing financial interests. 